九州大学学術情報リポジトリ

Kyushu University Institutional Repository

\title{
Revision of the Dissolution Kinetics of Aggregated Settling Particles
}

Akagi, Tasuku

Department of Earth and Planetary Sciences, Faculty of Sciences, Kyushu University

https://doi.org/10.5109/1397628

出版情報 : 九州大学大学院理学研究院紀要 : Series D, Earth and planetary sciences. 33 (1)，pp.15, 2013-12. Faculty of Science, Kyushu University バージョン：

権利関係 : 
Mem. Fac. Sci., Kyushu Univ., Ser. D, Earth \& Planet. Sci., Vol. XXXIII, No. 1, pp. 1-5, December 00, 2013

\title{
Revision of the Dissolution Kinetics of Aggregated Settling Particles
}

\author{
Tasuku Akagi
}

\begin{abstract}
The dissolution kinetics theory of settling particles, which was developed to express the elemental concentration variation as a function of diatom productivity, is revised to allow further consideration on the presence of voids. In this revision, the number of diatom frustules which form an aggregate is introduced as a basic variable to secure the rigidity of mathematical consideration, (unlike the previous paper, where productivity is employed as one of the basic variations - Akagi et al., 2011). No exchange of elements is assumed between seawater surrounding the aggregates and seawater in the voids of the aggregates. It is shown that the presence of voids reduces the settling velocity, but does not affect the dissolution/alteration behavior of settling particles as long as the particles are collected at a certain depth.
\end{abstract}

Keywords: settling particles, aggregate, dissolution theory, voids

\section{Introduction}

Interaction between particles and solution is one of the most important processes to control the distribution and behavior of dissolved matter in the oceans. This interaction includes chemically describable reactions such as dissolution, desorption, adsorption, etc. However, independent movements of particles against the medium make a seawater column a complex system. I believe that this is one of the reasons for rather inconsistent compositional data of the settling particles (Archer, 2006), which have hampered a clear understanding of the interaction.

Akagi et al. (2011) observed that the composition of settling particles always exhibits a hyperbolic relationship against opal mass flux $(P)$ and proposed the dissolution kinetics of diatom opal, as expressed by eq. (1).

$$
C_{i} \approx C_{i}^{o}\left(1-\frac{k_{\mathrm{d}, i} D}{P^{2}}\right),
$$

where $C_{i}, D$ and $k_{\mathrm{d}, i}$ are concentration of the element $(i)$ in particles, depth the particles are sampled at, and a dissolution constant of $i$, respectively. $C_{i}{ }^{0}$ indicates the concentration of $i$, before dissolution. I believe that the dissolution kinetics is the first successful consistent interpretation of the compositional variation of settling particles. In the kinetics diatom frustules aggregate to form larger particles, which transfer through a water column with a smaller length of time and a lesser proportion of exposed surface to contact with seawater. As a result of decreasing time and surface contact with seawater, the aggregates tend to retain their original composition with a lesser extent of dissolution/alteration. Akagi et al. (2011) then concluded that the asymptotic values of the hyperbolic relationships correspond to the original values of frustules $\left(C_{i}^{0}\right.$ in eq (1)). This has later been verified by the diatom incorporation theory (Akagi, 2013). In the model the settling velocity and the extent of dissolution/alteration are related through the size of aggregates. However, the model does not explicitly include the number of frustules in aggregates in the derivation of the model equations, rendering further consideration rather difficult.

Naturally observed marine snows show variation in density (Alldredge and Gotschalk, 1988), and voids of varying proportion may be present in real aggregates. It is likely that the aggregates gradually lose voids and

Manuscript received on 25 September 2013; accepted on 6 November 2013

Department of Earth and Planetary Sciences, Faculty of Sciences, 33 Kyushu University, 6-10-1 Hakozaki, Higashi-ku, Fukuoka 812-8581, Japan 
become denser during settlement to increase settling velocities (Berelson, 2002). In this paper, the model is modified to incorporate the number of frustules and the effect of presence of voids on the dissolution kinetics of aggregates is theoretically considered.

\section{Dissolution Kinetics Theory of a Single Aggregated Particle}

Let $m_{i}$ be mass of an element, $i$, in a single particle. Following the dissolution kinetics (Akagi et al., 2011), dissolution, $d m_{i}$, is proportional to both contact time $d t$ and surface area:

$$
d m_{i}=k_{i} C_{i} S d t
$$

where $k_{i}, C_{i}$ and $S$ are the proportionality constant, concentration of the element in the particle, and surface area, respectively. Adsorption reaction may follow a slightly different equation, but nonetheless similar to eq. (2) with the opposite sign of $k_{i}$ and general term of dissolution/alteration can be applied to refer to the equation. The length of time the particle is in contact with seawater is proportional to the distance that the particle travels, D, and the reciprocal of the settling velocity, $v$. Therefore,

$$
\Delta m_{i}=k_{i} C_{i} S \frac{D}{v} .
$$

Here it is shown how the three parameters, $C_{i}, S$ and $v$, are expressed as a function of the volume proportion of frustules, $f$, in an aggregated particle consisting of a number of diatom frustules, $n$ (see Fig. 1). Each frustule has a unit volume of $V_{\mathrm{p}}$ and density $\rho_{\mathrm{p}}$. The volume of the particle, $V$, is expressed by

$V=\frac{n V_{\mathrm{p}}}{f}$.

Assuming the particle is spherical, the radius, $r$, and surface area, $S$, of the particles are

$r=\left(\frac{3 V}{4 \pi}\right)^{\frac{1}{3}}=\left(\frac{3 n V_{\mathrm{p}}}{4 \pi f}\right)^{\frac{1}{3}}$

and

$$
S=4 \pi\left(\frac{3 n V_{\mathrm{p}}}{4 \pi f}\right)^{\frac{2}{3}},
$$

respectively.

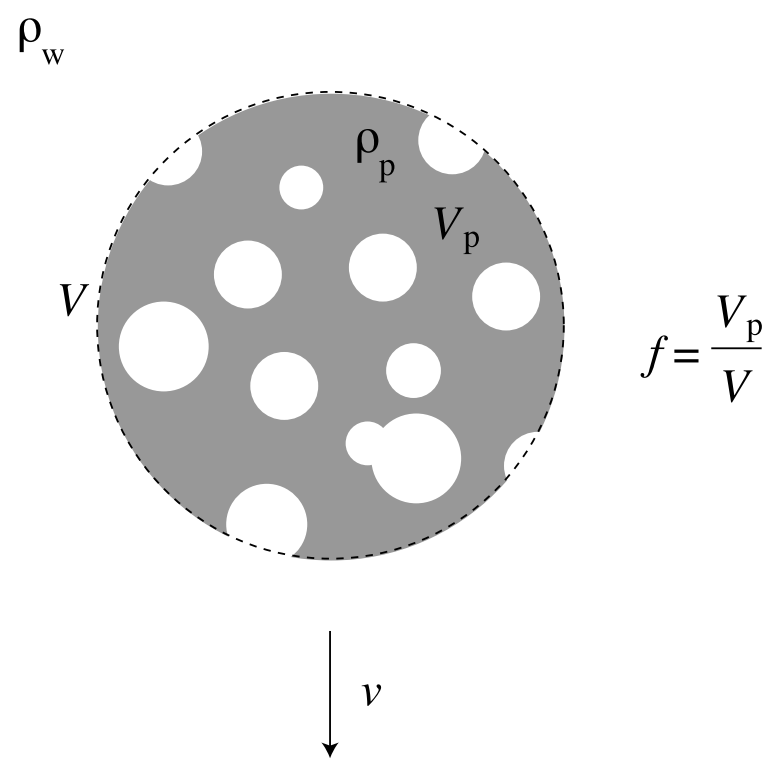

Fig. 1 Schematic diagram of a settling aggregated particle with voids of an aqueous medium. 
The volume proportion of voids in the particle is $1-f$. If the voids are filled with seawater of density $\rho_{\mathrm{w}}$, the density of the particle, $\rho$, is

$\rho=f \rho_{\mathrm{p}}+(1-f) \rho_{\mathrm{w}}=f\left(\rho_{\mathrm{p}}-\rho_{\mathrm{w}}\right)+\rho_{\mathrm{w}}$.

According to Stokes' law, settling velocity of the particles is

$v=k\left(\rho-\rho_{\mathrm{w}}\right) r^{2}=k f\left(\rho_{\mathrm{p}}-\rho_{\mathrm{w}}\right)\left(\frac{3 n V_{\mathrm{p}}}{4 \pi f}\right)^{\frac{2}{3}}$,

where $k$ is a proportionality constant.

The concentration $C_{i}$ of the particles is also diluted by the voids, i.e.,

$C_{i}=f C_{i, \mathrm{p}}$.

Putting eqs. (6), (8) and (9) into eq. (3), we have

$$
\Delta m_{i}=k_{i} \frac{f C_{i, \mathrm{p}} 4 \pi\left(\frac{3 n V_{\mathrm{p}}}{4 \pi f}\right)^{\frac{2}{3}} D}{k f\left(\rho_{\mathrm{p}}-\rho_{\mathrm{w}}\right)\left(\frac{3 n V_{\mathrm{p}}}{4 \pi f}\right)^{\frac{2}{3}}}=\frac{k_{i} C_{i, \mathrm{p}} D}{k\left(\rho_{\mathrm{p}}-\rho_{\mathrm{w}}\right)} .
$$

It should be noted that the proportion of frustules in the aggregate, $f$, and surface area, $S$, are both eliminated in eq. (10).

Since the mass of the aggregate is $n V_{\mathrm{p}} \rho_{\mathrm{p}}$, the concentration of the element after a certain time is expressed as

$$
n V_{\mathrm{p}} \rho_{\mathrm{p}} C_{i}=n V_{\mathrm{p}}^{o} \rho_{\mathrm{p}}^{o} C_{i}^{o}-\Delta m_{i},
$$

where superscript $o$ indicates the initial values. When the change is small, the equation can be approximated to

$$
C_{i} \approx C_{i}^{o}\left(1-\frac{\Delta m_{i}}{n V^{o} \rho_{\mathrm{p}}^{o} C_{i}^{o}}\right)
$$

Therefore,

$$
C_{i} \approx C_{i}^{o}\left(1-\frac{K_{i} D}{n}\right)
$$

$K$ is an overall proportionality constant,

$$
K_{i}=\frac{k_{i}}{k\left(\rho_{\mathrm{p}}^{o}-\rho_{\mathrm{w}}\right) V^{o} \rho_{\mathrm{p}}^{o}} .
$$

Eqs. (1) and (13) are identical to each other if $n$ is proportional to $P^{2}$. The number of frustules to aggregate, $n$, is a function of frustule density, but it should be noted that $n$ is not a function of productivity, $P$. (Note $P$ is expressed by mass of product per unit area per unit time and is different from the density in dimension.) It is often the case that the number of frustules to aggregate, $n$, tend to increase with an increase of $P$. This rather vague remark on $n-P$ linkage is enough to explain the observed hyperbolic relationship between the concentration of elements in settling particles and productivity (Akagi et al., 2011). This also provides the rationale that one can obtain the original concentration of frustules when $P$ is great enough (Akagi et al., 2011). 


\section{Results and Discussion}

\subsection{Influence of voids in the dissolution of elements in aggregates}

In eq. (13) $f$ is eliminated. It is thus concluded that, as long as settling particles are sampled at a fixed depth, the extent of dissolution is not affected by the change in the proportion of voids. In the model no exchange of elements is assumed between seawater surrounding the aggregates and seawater in the voids of the aggregates. If aggregates are packed enough, this assumption may be considered to be valid.

\subsection{Influence of voids in settling velocity of aggregates}

Eq. (8) indicates that the settling velocity is proportional to $f^{1 / 3}$. Berelson (2002) observed the increase in settling velocities of particles with increasing depth. This consideration suggests that the decrease of a void volume with increasing depth may be one of the reasons.

\section{Conclusion}

The properties of settling particles theoretically derived by this study are summarized in Fig. 2 . The presence of voids in settling particles increases particle size and the density of the particles becomes closer to the medium, compared with void-free settling particles. The settling velocity becomes lower as the results of both effects. Because the settling particles are normally collected using a sediment trap deployed at a certain depth, it is found that the dissolution/alteration of the particles during the settlement of a water column is not affected by the presence of voids and the same equation used in void-free particles expresses the dissolution of elements in a water column. The present model may show that one of the most important factors to determine dissolution/ alteration of aggregates is the number of frustules to form aggregates, but not the presence of voids. The presence of voids allows some less-altered highly-aggregated particles to settle at the same rate as highly-altered lessaggregated particles.

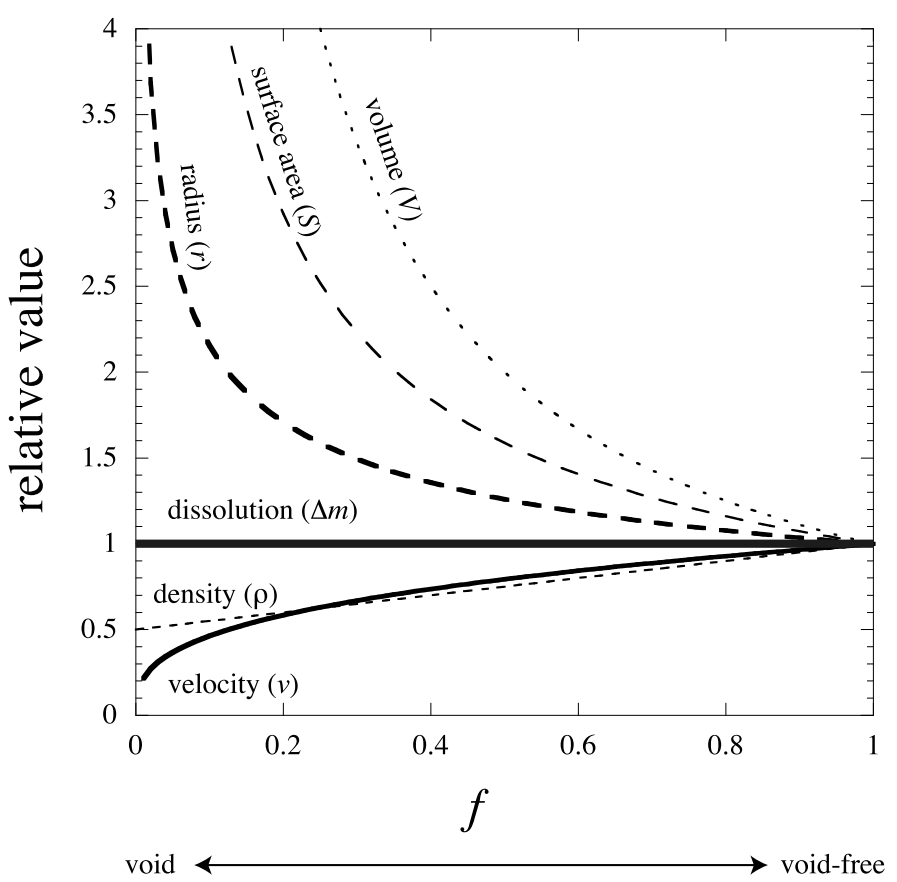

Fig. 2 Theoretical relationships of volume, surface area, particle size, density, settling velocity, dissolution/alteration extent of a single aggregated particle v.s. the presence of voids $(1-f)$. Values are relative to the corresponding ones of the void-free aggregated particle $(f=1)$. In the calculation $\rho_{\mathrm{p}}$ is set to $2 \rho_{\mathrm{w}}$. 
Acknowledgement: I thank Dr Yusuke Okazaki for his comments on the manuscript. This research was supported by MEXT/JSPS Grant Number 23310008.

\section{References}

Akagi, T., Fu F.-F., Hongo, Y. \& Takahashi, K. (2011) Composition of Rare Earth Elements in Settling Particles Collected in the Highly Productive North Pacific Ocean and Bering Sea: Implications for Siliceous-Matter Dissolution Kinetics and Formation of Two REE-Enriched Phases. Geochim. Cosmochim. Acta, 75, 48574876.

Akagi, T. (2013) Rare earth element (REE)-silicic acid complexes in seawater to explain the incorporation of REEs in opal and the "leftover" REEs in surface water: new interpretation of dissolved REE distribution profiles. Geochim. Cosmochim. Acta, 113, 174-192.

Alldredge A.L. and Gotschalk C. (1988) In situ settling behavior of marine snow. Limnol. Oceanogr., 33, 339-351.

Anderson R.F. (2006) Chemical tracers of particle transport. In H. Elderfield(ed.) Treatise on Geochemistry. Vol. 6 The Oceans and Marine Geochemistry. Elsevier, 247-273.

Archer D. (2006) Biological fluxes in the ocean and atmospheric $\mathrm{pCO}_{2}$. In H. Elderfield(ed.) Treatise on Geochemistry. Vol. 6 The Oceans and Marine Geochemistry. Elsevier, 275-291.

Berelson W. M. (2002) Particle settling rates increase with depth in the ocean. Deep-Sea Res. II, 49, 237-251. 